Nota de las Editoras

Editors' Notes

Lettre des éditeurs

Nota de les Editores

DOI: https://dx.doi.org/10.5565/rev/jt13.745

\title{
Nota de las Editoras
}

Como siempre es un placer anunciar la publicación de otro volumen de BJTLLL. En un momento en que el mundo de la publicación académica se enfrenta a bastantes polémicas, entre las cuales está la inequidad que surge del uso creciente de las métricas como medida de la calidad de la investigación, seguimos manteniendo nuestro compromiso de publicar nuevas investigaciones prometedoras de jóvenes que están empezando a profundizar en sus respectivos campos, manteniendo al mismo tiempo un alto nivel de calidad de su producción. Siguiendo estas líneas, este volumen contiene tres artículos científicos estimulantes e innovadores llevados a cabo por jóvenes investigadores en los campos de la enseñanza y del aprendizaje de la lengua y de la literatura.

Comenzamos este tema con nuestros autores invitados Balaman y Sert, dos investigadores muy reconocidos dentro del área de Análisis de la Conversación. Su artículo incluye un análisis detallado de las competencias interaccionales de un alumno interactuando en su L2 dentro de dos contextos diferentes: el primero en una tarea de discusión cara a cara y el segundo en una tarea "on-line" (utilizando medios de comunicación digitales). La discusión y los resultados con respecto a los turnos de conversación, la asignación de turnos y la gestión de las acciones dentro de la interacción proporcionan una importante perspectiva en un área creciente de la investigación de la enseñanza de la lengua: el diseño de las tareas de aprendizaje en contextos cara a cara y "on-line".

El artículo de los autores invitados continúa con un estudio de un caso sobre las creencias, representaciones y conocimientos de los profesores de idiomas en relación a la perspectiva de la evaluación plurilingüe en un contexto escolar internacional (escrito por Mate). A continuación, Casañ Núñez describe el diseño y luego explica los resultados de una investigación multi-fase de métodos mixtos sobre ejercicios de comprensión auditiva / visual, facilitados a través de preguntas de comprensión audiovisuales integradas en secuencias de vídeo subtituladas. Nuestro último artículo final de esta sección, escrito por Ugwuanyi, explica un estudio de métodos mixtos a gran escala de dos grupos de estudiantes de la Universidad de Nigeria que fueron enseñados desde dos enfoques diferentes. Un grupo fue enseñado a través de un enfoque basado en la literatura, mientras que el otro grupo fue instruido a través de un enfoque basado en la gramática. Basándose en las conclusiones extraídas de los resultados, el autor ofrece recomendaciones para la enseñanza de lenguas a través de un enfoque naturalista. Existen varios nexos concomitantes entre los artículos de esta sección, comenzando por las conexiones directas o indirectas con el tema de la evaluación del aprendizaje de idiomas, ya sea que se considere desde la perspectiva del profesor o del rendimiento del estudiante. Esta es una cuestión clave en la educación lingüística y literaria y se ha destacado en la excelente investigación realizada por los investigadores publicados aquí.

En la sección de reseña de libros, Calvo Benzies discute el libro recientemente publicado titulado Enseñanza y Aprendizaje de Lenguas Extranjeras en Educación Secundaria y 
Obligatoria, editado por Dolors Masats y Luci Nussbaum. Para cerrar este volumen, nos complace ofrecer al lector una entrevista con el Dr. José Plácido Ruiz Campillo, Profesor Titular del Departamento de Culturas Iberoamericanas de la Universidad de Columbia en Nueva York. Sánchez-Jiménez discute con el Dr. Ruiz Campillo aspectos de la gramática operativa cognitiva y su impacto en la enseñanza del español como lengua extranjera.

Esperamos que los textos sean inspiradores para futuras investigaciones e innovaciones.

Dra. Melinda Dooly y Dra. Emilee Moore

30 de septiembre de 2017

\section{Créditos:}

Las ilustraciones de las portadas de cada número han sido diseñadas por estudiantes de EINA (Escola de Disseny i Art, Barcelona), que cursan el posgrado Il·lustració Creativa, bajo la dirección de Sonia Pulido, profesora de Ilustración para Prensa.

\section{Revisores para el Volumen 10.3}

Las editoras desean agradecer a los siguientes revisores su contribución a la preparación del Volumen 10.3:

Jesús Alirio Bastidas, Universidad de Nariño; Josep Maria Cots, Universitat de Lleida; Anna Cros, Universitat Autònoma de Barcelona; Nicolas Guichon, Université Lyon II/Université du Québec à Montréal; Dolors Masats, Universitat Autònoma de Barcelona, Josep Soler Carbonell, Stockholms Universitet, y Lou Tolosa, University of Georgia. 


\section{Editors' Notes}

As always it is our pleasure to announce the publication of another volume of BJTLLL. At a time when academic publishing is becoming rife with polemic issues, not least of which is inequity that arises from the increasing use of metrics as a measure of research quality, we continue to uphold our commitment to publishing promising new research of young scholars breaking into their respective fields while maintaining a high level of quality of output. Along these lines, this issue has three exciting and innovative research articles by new researchers in the fields of language and literature teaching and learning.

We begin this issue with our invited authors Balaman and Sert, two well-known researchers working in the area of Conversation Analysis and online data. The article discusses a detailed analysis of the interactional competencies, complemented with a wide variety of interactional resources, that a focal L2 learner deploys in two contexts: a face-to-face discussion task and an online emergent information gap task. The discussion and findings in regards to turn taking, allocation and action formation provide insight into a growing area of language teaching research: task design in face-to-face and online settings.

This article is followed by Mate's case study on the beliefs, representations and knowledge of language teachers in regards to plurilingual evaluation approaches in an international school context. Next, Casan describes and discusses a multiphase mixed-methods investigation into listening/viewing comprehension exercises facilitated through audiovisual comprehension questions embedded within a subtitled video sequences. Our final article in this section, written by Ugwuanyi, discusses a large-scale mixed methods study of two groups of students at the University of Nigeria who were taught using two different approaches. One group was taught through a literature-based approach while the other group was instructed through a grammar-based focus. Based on the conclusions drawn from the results, the article provides recommendations for teaching through a Naturalist Approach to language. There are several concomitant nexus that run throughout the articles beginning with direct or indirect connections to the issue of evaluating language learning, whether it is looked at from the teacher's perspective, or student performance. This is a key issue in language and literature education and has been highlighted in the excellent research carried out by the researchers published here.

Both the book review fits appropriately into the general theme of articles included in this volume. Calvo Benzies reviews the recently published book entitled Enseñanza y Aprendizaje de Lenguas Extranjeras en Educación Secundaria y Obligatoria, edited by Dolors Masats and Luci Nussbaum. To close this volume, we are pleased to offer the reader with an interview of Dr. José Plácido Ruiz Campillo, Senior Lecturer at the Department of Latin American and Iberian Cultures of Columbia University In New York. Sánchez-Jiménez discusses with Dr. Ruiz Campillo aspects of cognitive-based operative grammar and its impact on the teaching of Spanish as a foreign language.

As always, we hope you find the texts to be inspiring for future research and innovation. Dr. Melinda Dooly \& Dr. Emilee Moore

30 September 2017 


\section{Credits:}

Illustrations for the covers of each issue are designed by students of EINA (Escola de Disseny i Art, Barcelona) studying in the postgraduate course 'Il-lustració Creativa', under the direction of Sonia Pulido, teacher of Illustration for Publishing Media.

\section{Reviewers for Volume 10.3}

The editors would like to thank the following reviewers for their contribution to the preparation of Volume 10.3:

Jesús Alirio Bastidas, Universidad de Nariño; Josep Maria Cots, Universitat de Lleida; Anna Cros, Universitat Autònoma de Barcelona; Nicolas Guichon, Université Lyon II/Université du Québec à Montréal; Dolors Masats, Universitat Autònoma de Barcelona, Josep Soler Carbonell, Stockholms Universitet, \& Lou Tolosa, University of Georgia. 


\section{Lettre des éditeurs}

Comme toujours, c'est un plaisir d'annoncer la publication d'un autre volume de BJTLLL. À un moment où le monde de l'édition académique fait face à beaucoup de controverse, parmi lesquelles l'inégalité découlant de l'utilisation croissante des indicateurs comme mesure de la qualité de la recherche, nous continuons à maintenir notre engagement à publier de nouvelles recherches prometteuses de les jeunes qui commencent à approfondir dans leurs domaines respectifs, tout en conservant un niveau élevé de qualité de leur production. Dans ce sens, ce volume contient trois articles scientifiques stimulants et novateurs réalisés par de jeunes chercheurs dans les domaines de l'enseignement et de l'apprentissage du langage et de la littérature.

Nous commençons ce volume avec nos auteurs invités Balaman et Sert, deux chercheurs bien connus dans le domaine de l'analyse de la conversation. Son article comprend une analyse détaillée des compétences interactives d'un élève qui interagit dans son L2 dans deux contextes différents: le premier dans une tâche de discussion en face à face et le second dans une tâche en ligne (à l'aide de la technologie de communication). La discussion et les résultats concernant les conversations par tournes, la répartition des tâches et la gestion des actions au sein de l'interaction fournissent une perspective importante dans un domaine émergent de recherche en enseignement des langues: la conception des tâches d'apprentissage en face-àface et en ligne.

L'article des auteurs invités se poursuit avec une étude de cas sur les croyances, les représentations et la connaissance des enseignants de langues par rapport à la perspective de l'évaluation multilingue dans un contexte scolaire international (écrit par Mate). Ensuite, Casañ Núñez décrit le design et explique ensuite les résultats d'une étude multiphasée de méthodes mixtes sur les exercices d'écoute / compréhension visuelle, facilitée par des questions de compréhension audio-visuelle intégrées dans des séquences vidéo sous-titrées. Notre dernier article final dans cette section, écrit par Ugwuanyi, explique une étude mixte à grande échelle de deux groupes d'étudiants de l'Université du Nigéria qui ont enseigné à partir de deux approches différentes. Un groupe a été enseigné dans le cadre d'une approche basée sur la littérature, tandis que l'autre groupe a été instruit par une approche basée sur la grammaire. Sur la base des conclusions tirées des résultats, l'auteur propose des recommandations pour l'enseignement des langues par une approche naturaliste. Il existe plusieurs liens concomitants entre les articles de cette section, en commençant par des connexions directes ou indirectes au sujet de l'évaluation de l'apprentissage des langues, qu'elles soient vues du point de vue de l'enseignant ou de la performance des élèves. C'est une question clé dans l'éducation linguistique et littéraire et a été souligné dans l'excellente recherche menée par les chercheurs publiés ici.

Dans la section de revue de livres, Calvo Benzies discute du livre récemment publié intitulé Enseñanza y Aprendizaje de Lenguas Extranjeras en Educación Secundaria y Obligatoria, édité par Dolors Masats et Luci Nussbaum. Pour fermer ce volume, nous sommes ravis d'offrir au lecteur une interview avec le Dr José Plácido Ruiz Campillo, professeur titulaire du Département des Cultures Ibéro-Américaines de l'Université Columbia à New York. SánchezJiménez discute avec Dr. Ruiz Campillo des aspects de la grammaire cognitive opérationnelle et son impact sur l'enseignement de l'espagnol comme langue étrangère.

Nous espérons que les textes inspireront la recherche et l'innovation futures. 
Dr Melinda Dooly \& Dr Emilee Moore

30 septembre 2017

\section{Crédits:}

Les illustrations des couvertures de chaque numéro sont élaborées par des étudiants d'EINA (École de Design et d'Art, Barcelone) réalisant le cours de Post-grado « Illustration créative », sous la direction de Sonia Pulido, professeur d'illustration pour médias imprimés.

\section{Réviseurs du Volume 10.3.}

Les éditeurs voudraient remercier les réviseurs suivants pour leur contribution à la préparation du Volume 10.3:

Jesús Alirio Bastidas, Universidad de Nariño; Josep Maria Cots, Universitat de Lleida; Anna Cros, Universitat Autònoma de Barcelona; Nicolas Guichon, Université Lyon II/Université du Québec à Montréal; Dolors Masats, Universitat Autònoma de Barcelona, Josep Soler Carbonell, Stockholms Universitet, et Lou Tolosa, University of Georgia. 


\section{Nota de les Editores}

Com sempre és un plaer anunciar la publicació d'un altre volum de BJTLLL. En un moment en què el món de la publicació acadèmica s'enfronta a bastants polèmiques, entre les quals hi ha la inequitat que sorgeix de l'ús creixent de les metriques com a mesura de la qualitat de la investigació, seguim mantenint el nostre compromís de publicar noves promeses de recerca de joves que estan començant a aprofundir en els seus respectius camps, mantenint al mateix temps un alt nivell de qualitat de la seva producció. Seguint aquestes línies, aquest volum conté articles científics estimulants i innovadors portats a terme per joves investigadors en els camps de l'ensenyament i de l'aprenentatge de la llengua $i$ de la literatura.

Comencem aquest tema amb els nostres autors convidats Balaman i Sert, dos investigadors molt reconeguts dins l'àrea d'Anàlisi de la Conversa. El seu article inclou una anàlisi detallada de les competències interaccionals d'un alumne interactuant en el seu L2 dins de dos contextos diferents: el primer en una tasca de discussió cara a cara i el segon en una tasca "en línia" (utilitzant mitjans de comunicació digitals ). La discussió i els resultats amb respecte als girs de conversa, l'assignació de torns i la gestió de les accions dins de la interacció proporcionen una important perspectiva en una àrea creixent de la recerca de l'ensenyament de la llengua: el disseny de les tasques l'aprenentatge en contextos cara a cara i "en línia".

L'article dels autors convidats continua amb un estudi sobre un cas sobre les creences, representacions i coneixements dels professors d'idiomes en relació amb la perspectiva de l'avaluació plurilingüe en un context escolar internacional. A continuació, Casañ Núñez descriu el disseny i després explica els resultats d'una investigació multifase de mètodes mixtos sobre exercicis d'entesa auditiva / visual, facilitats a través de preguntes d'enteniment audiovisual integrades en seqüències de vídeo subtitulades. El nostre últim article final d'aquesta secció, escrit per Ugwuanyi, explica un estudi de mètodes mixtos a gran escala de dos grups d'estudiants de la Universitat de Nigèria que van ser ensenyats des de dos enfocaments diferents. Un grup va ser ensenyat a través d'un enfocament basat en la literatura, mentre que l'altre grup va ser instruït a través d'un enfocament basat en la gramàtica. Basantnos en les conclusions extretes dels resultats, l'autor ofereix recomanacions per a l'ensenyament de llengües a través d'un enfocament naturalista. Existeixen diversos nexos concomitants entre els articles d'aquesta secció, començant per les connexions directes o indirectes amb el tema de l'avaluació de l'aprenentatge d'idiomes, ja sigui que es consideri des de la perspectiva del professor o del rendiment de l'estudiant. Aquesta és una qüestió clau en l'educació lingüística i literària i s'ha destacat en l'excel·lent recerca realitzada pels investigadors publicats aquí.

En la secció de ressenya de llibres, Calvo Benzies discuteix el llibre recentment publicat titulat Enseñanza y Aprendizaje de Lenguas Extranjeras en Educación Secundaria y Obligatoria, editat per Dolors Masats i Luci Nussbaum. Per tancar aquest volum, ens complau oferir al lector una entrevista amb el Dr. José Plácido Ruiz Campillo, Professor Titular del Departament de Cultures Iberoamericanes de la Universitat de Columbia a Nova York. Sánchez-Jiménez discuteix amb el Dr. Ruiz Campillo aspectes de la gramàtica operativa cognitiva i el seu impacte en l'ensenyament de l'espanyol com a llengua estrangera.

Com sempre, esperem que els textos siguin inspiradors per a futures investigacions i innovacions. 
Dra. Melinda Dooly i Dra. Emilee Moore

30 de setembre de 2017

\section{Crèdits:}

Les il·lustracions per a les portades de cada número estan dissenyades pels estudiants d'EINA (Escola de Disseny i Art, Barcelona) que estudien en el curs de postgrau Il·lustració Creativa, sota la direcció de Sonia Pulido, professora d'Il·lustració per a Premsa.

\section{Revisors del Volum 10.3}

Les editores volen agrair als següents revisors la seva contribució a la preparació del Volum 10.3:

Jesús Alirio Bastidas, Universidad de Nariño; Josep Maria Cots, Universitat de Lleida; Anna Cros, Universitat Autònoma de Barcelona; Nicolas Guichon, Université Lyon II/Université du Québec à Montréal; Dolors Masats, Universitat Autònoma de Barcelona, Josep Soler Carbonell, Stockholms Universitet, i Lou Tolosa, University of Georgia.

Per citar aquest article:

Dooly, M., \& Moore, E. (2017). Nota de las editoras, 10.3. Bellaterra Journal of Teaching \& Learning Language \& Literature, 10(3), 1-8. DOI: http://dx.doi.org/10.5565/rev/jt13.745 